\title{
First Principles Studies on the Electronic Structure and Band Structure of Paraelectric $\mathrm{SrTiO}_{3}$ by Different Approximations
}

\author{
Hamdollah Salehi \\ Department of Physics, University of Shahid Chamran, Ahvaz, Iran \\ E-mail:salehi_h@scu.ac.ir \\ Received April 3, 2011; revised May 29, 2011; accepted June 16, 2011
}

\begin{abstract}
The electronic structure, energy band structure, total density of states (DOS) and electronic density of perovskite $\mathrm{SrTiO}_{3}$ in the cubic phase are calculated by the using full potential-linearized augmented plane wave (FP-LAPW) method in the framework density functional theory (DFT) with the generalized gradient approximation (GGA) by WIEN2k package. The calculated band structure shows a direct band gap of $2.5 \mathrm{eV}$ at the $\Gamma$ point in the Brillouin zone.The total DOS is compared with experimental x-ray photoemission spectra. From the DOS analysis, as well as charge-density studies, I have conclude that the bonding between $\mathrm{Sr}$ and $\mathrm{TiO}_{2}$ is mainly ionic and that the $\mathrm{TiO}_{2}$ entities bond covalently. The calculated band structure and density of state of $\mathrm{SrTiO}_{3}$ are in good agreement with theoretical and experimental results.
\end{abstract}

Keywords: Electronic Structure, $\mathrm{SrTiO}_{3}$, WIEN2k, FP-LAPW, DFT, GGA

\section{Introduction}

Ferroelectric and related materials having chemical formula $\mathrm{ABO}_{3}$ have been the subject of extensive investigation, both because of their technical importance and because of the fundamental interest in the physics of their phase transitions [1]. Within this family of materials, one finds transitions to a wide variety of low-symmetry phases, ranging from non-polar antiferrodistortive to polar ferroelectric and antiferroelectric transitions. The ideal structure is cubic perovskite, where the A and B cations are arranged on a simple cubic lattice and the $\mathrm{O}$ ions lie on the face centers nearest the (typically transition metal) $\mathrm{B}$ cations. Thus the $\mathrm{B}$ cations are at the center of $\mathrm{O}$ octahedral, while the A cations lie at larger twelvefold-coordinated sites. This ideal structure displays a wide variety of structural instabilities in the various materials. These may involve rotations and distortions of the $\mathrm{O}$ octahedra as well as displacements of the cations from their ideal sites. The interplay of these instabilities accounts for the rich variety of ferroelectric and antiferroelectric behaviours. $\mathrm{SrTiO}_{3}$ in this class has been the subject of ongoing theoretical and experimental studies because of its unusual dielectric property which deviates from those of other $\mathrm{ABO}_{3}$ perovskites. While it has the simple cubic structure at high temperature, $\mathrm{SrTiO}_{3}$ goes through an AFD transition at $105 \mathrm{~K}$ to a tetragonal phase in which the oxygen octahedra have rotated by a smal angle along the c-axis in opposite senses in neighbouring unit cells. These titanates have recently attracted the attention of many researchers because some titanates are practically used as ferroelectric, electroconductive, photorefractive and photovoltaic materials. It is well known that these applications are based on electrical and optical properties of the materials, and the properties result from their electronic structures. Therefore, an understanding of the electronic structure of the materials is fairly important to improve the electrical or optical functions for their new applications. Investigations have been carried out to study the structural [2], dielectric [3], optical [4,5] and elastic [6] properties, and the infrared [7,8] as well as electron paramagnetic resonance [9] spectra of $\mathrm{SrTiO}_{3}$. Although various properties [10-15] of $\mathrm{SrTiO}_{3}$ have been investigated, a systematic theoretical study of the optical properties based upon first-principles band structure calculations is still lacking. In the present study, the electronic structure, density of state and electronic density of $\mathrm{SrTiO}_{3}$ in the paraelectric phase are calculated by the FPLAPW method with the DFT in GGA [16,17] Recently, king-Smith and Vanderbilt performed a systematic study 
of structural and dynamical properties and energy surfaces for eight common perovskites, using the first-principles ultrasoft-pseudopotential method and the density approximation (LDA) [18,19]. Finally, the calculated results are compared with available experimental data $[5,20]$.

\section{The Calculation Method}

The paraelectric phase of $\mathrm{SrTiO}_{3}$ has the ideal cubic pm3m perovskite structure, in which the oxygen octahedron contain a $\mathrm{Ti}$ atom at its center. The calculations have been performed considering the origin of the cell to be at the Sr site, Ti at the body-center $(0.5,0.5,0.5) \mathrm{a}$ and the three $\mathrm{O}$ atoms at the three face centres $(0.5,0.5,0.0) \mathrm{a}$, $(0.0,0.5,0.5) \mathrm{a}$ and $(0.5,0.0,0.5) \mathrm{a}$. The lattice constant is 7.38a.u, taken from the experimental results of Wyckoff [21]. Figure 1(a) shows the unit cell and Figure 1(b) the Brilluoin zone of $\mathrm{SrTiO}_{3}$ for this structure.

The electronic structure, band structure, DOS and electronic density in cubic crystal $\mathrm{SrTiO}_{3}$ are studied using the Wien2k package [22]. This employs the FP-LAPW method in the framework of the DFT with the GGA for solving the kohn-Sham equation [16,17]. In this method no shape approximation on either potential or the electronic charge density is made. In this method valence and core states origin energy have been separate, and $-6 \mathrm{Ry}$ energy bound separated between valence electrons and

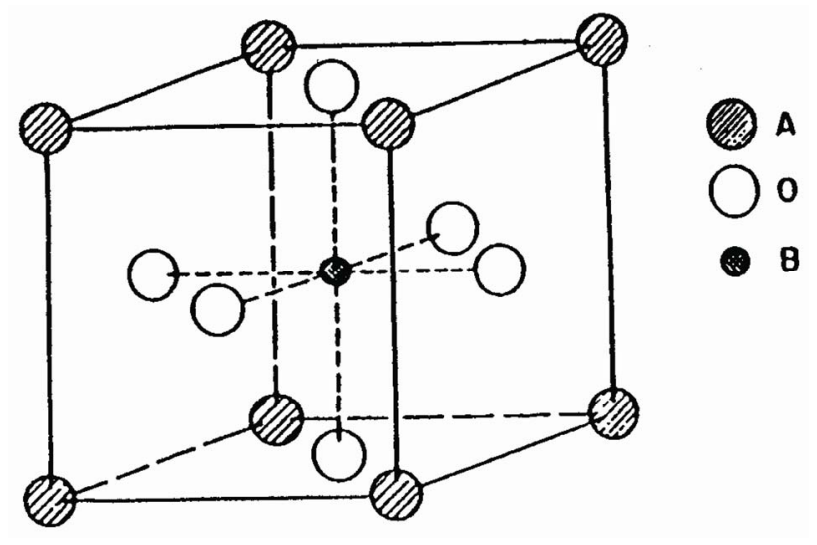

(a)

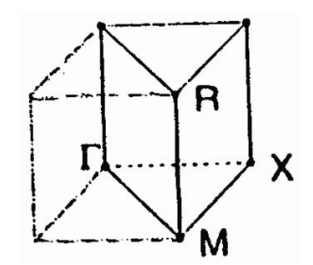

(b)

Figure 1. (a) The cubic unit cell of $\mathrm{SrTiO}_{3}$. (b) The Brillouin zone for the cubic $\mathrm{SrTiO}_{3}$. core states was chosen. The convergence parameter $\mathrm{Rk}_{\max }$, which controls the size of the basis sets in these calculations, was set to 7.0. I used $7 \times 7 \times 7$ meshes which represent $400 \mathrm{k}$ points in the first Brillouin zone Integrations in reciprocal space Ire performed using the special points method. I used meshes which represent $400 \mathrm{k}$-points in the first BZ. This corresponds to 20 special k-points in the irreducible idge for the cubic structure.

\section{Results}

\subsection{Electronic Structure}

For calculating the lattic constant theoritically, the basestate energy of $\mathrm{SrTiO}_{3}$ is calculated for different volumes around balance valume of each case and partial changees around balance valume and method given. In this program, energy changes in valume are given by mornagone state equation. The total energy as a function of the volume for $\mathrm{SrTiO}_{3}$ is shown in Figure 2. Then, by given mornagone equation in different approximations lattice constant, bulk modulue, condensation, base - state energy of crystall and are calculated with LDA and GGA. The results are compared with other experimental and theoretical datas shown in Table 1.

Electronic structure shows in Figure 2 was calculated by using the lattice constant 7.38 a.u., taken from the experimental results of Wyckoff [21]. The distances between the nearest atoms are shown in Table 2 for cubic $\mathrm{SrTiO}_{3}$. These lengths for experimental and theoretical results, are equal, and do not depend on the exchange correlation potentials. The effective charges on the three $\mathrm{Sr}, \mathrm{Ti}$ and $\mathrm{O}$ atoms are shown in Table $\mathbf{3}$ and are very close to the value of the ionic charge.

\subsection{Band Structure}

The crystal structure of $\mathrm{SrTiO}_{3}$ in the paraelectric phase has been studied experimentally using various techniques $[3-5,8,19,23]$. This provides us with the structural parameters needed in our work and also the basis for comparison of the final results. Here in we calculations my used $400 \mathrm{k}$-points. The value for the convergence parameter taken to be, $\mathrm{Rk}_{\max }=7$. This is a parameter in the package that its right choice determines the stability and convergence of the calculations. The value of the parameter $\mathrm{Rk}_{\max }$ controls the size of the basis sets in these calculations. The value of $\mathrm{Rk}_{\max }$ for the systems studied, was chosen to be 7. Integrations in reciprocal space Ire performed using the special points method. I used kmeshes $7 \times 7 \times 7$ which represent $400 \mathrm{k}$-points in the first BZ. This corresponds to 20 special k-points in the irreducible Idge for the cubic structure. The calculated 


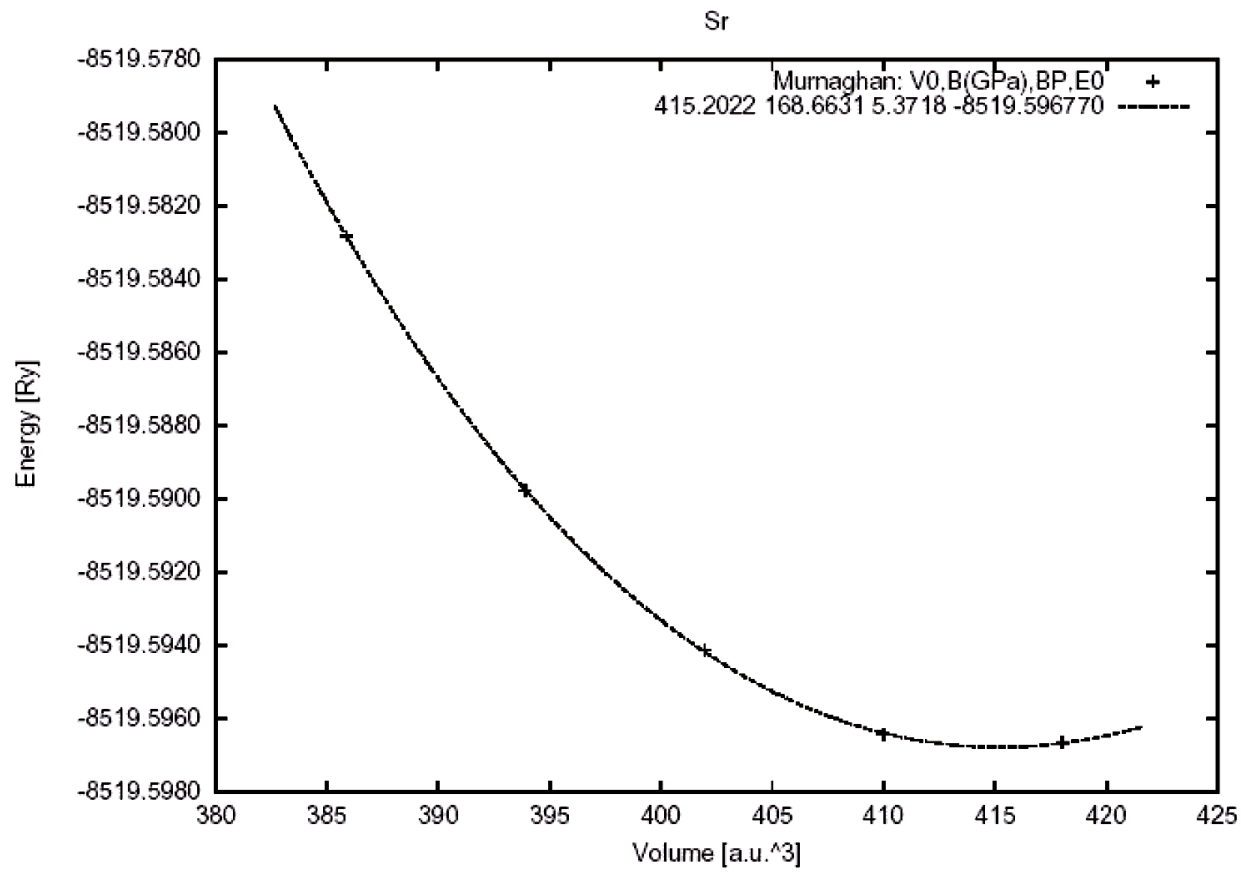

Figure 2. Total energy as a function of the volume for cubic $\mathrm{SrTiO}_{3}$.

Table 1. Structure parameters calculated in this work compared it with other results for cubic $\mathrm{SrTiO}_{3}$.

\begin{tabular}{cccccc}
\hline & FP-LAPW, & FP-LAPW & FP-LAPW, & Experimental & Theoretical \\
& GGA96 & GGA91 & LDA & {$[2,21]$} & LDA [18] \\
\hline A $(\mathrm{au})$ & 7.46 & 7.57 & 7.4 & 7.38 & 7 \\
$\mathrm{~B}(\mathrm{Gpa})$ & 168.7 & 165.13 & 130.42 & 183 & 173 \\
$\mathrm{~B}(\mathrm{Gpa})$ & 5.37 & 3.76 & 3.7 & - & - \\
$\mathrm{K}\left(\mathrm{m}^{2} / \mathrm{m}\right)$ & $5.93 \times 10^{-14}$ & $6.1 \times 10^{-14}$ & $7.7 \times 10^{-14}$ & $5.46 \times 10^{-14}$ & $5.8 \times 10^{-14}$ \\
$\mathrm{E}_{0}(\mathrm{Ry})$ & -8519.6 & -8520.991 & 8510.94 & - & - \\
\hline
\end{tabular}

Table 2. A compartion of the nearest distant between atoms for cubic $\mathrm{SrTiO}_{3}$.

\begin{tabular}{ccccccc}
\hline & $\begin{array}{c}\text { FP-LAPW, GGA96 } \\
\text { with Theoretical } \\
\text { Constant }\end{array}$ & $\begin{array}{c}\text { FP-LAPW, GGA96 } \\
\text { with experemintall } \\
\text { Constant }\end{array}$ & $\begin{array}{c}\text { FP-LAPW, GGA91 } \\
\text { with Theoretical } \\
\text { Constant }\end{array}$ & $\begin{array}{c}\text { FP-LAPW, GGA91 } \\
\text { with experemintall } \\
\text { Constant }\end{array}$ & $\begin{array}{c}\text { FP-LAPW, LDA } \\
\text { with Theoretical } \\
\text { Constant }\end{array}$ & $\begin{array}{c}\text { FP-LAPW, LDA } \\
\text { with experemintall } \\
\text { Constant }\end{array}$ \\
\hline Sr-O & 5.22 & 5.27 & 5.22 & 5.27 & 5.22 & 5.27 \\
Ti-O & 3.69 & 3.72 & 3.69 & 3.72 & 3.69 & 3.72 \\
\hline
\end{tabular}

Table 3. Effective charge calculated in this work compared other results for cubic $\mathrm{SrTiO}_{3}$.

\begin{tabular}{|c|c|c|c|c|c|c|c|}
\hline & $\begin{array}{c}\text { FP-LAPW, GGA96 } \\
\text { with Theoretical } \\
\text { Constant }\end{array}$ & $\begin{array}{c}\text { FP-LAPW, GGA96 } \\
\text { with experemintal } \\
\text { Constant }\end{array}$ & $\begin{array}{c}\text { FP-LAPW, GGA91 } \\
\text { with Theoretical } \\
\text { Constant }\end{array}$ & $\begin{array}{c}\text { FP-LAPW, GGA91 } \\
\text { with experemintall } \\
\text { Constant }\end{array}$ & $\begin{array}{c}\text { FP-LAPW, LDA } \\
\text { with Theoretical } \\
\text { Constant }\end{array}$ & $\begin{array}{c}\text { FP-LAPW, LDA } \\
\text { with experemintall } \\
\text { Constant }\end{array}$ & Formula \\
\hline $\mathrm{Sr}$ & 1.9123 & 1.9118 & 1.9131 & 1.9112 & 1.9126 & 1.9123 & +2 \\
\hline $\mathrm{Ti}$ & 2.0264 & 2.0234 & 2.0263 & 2.0197 & 2.0267 & 2.0202 & +4 \\
\hline $\mathrm{O}$ & 1.6923 & 1.6915 & 1.6920 & 1.6896 & 1.6835 & 1.6810 & -2 \\
\hline
\end{tabular}

electronic band structure of the cubic phase of $\mathrm{SrTiO}_{3}$ in the high symmetry directions in the Brillouin zone is shown in Figure 2. In the Figure 1 find a large dispersion of the bands. Nine valence bands are derived from $\mathrm{O}$
$2 p$ orbitals. These are separated by a direct gap $2.5 \mathrm{eV}$ (at the $\Gamma$-point) from the transition metal d-derived conduction band. Figure 3 shows that the FP-LAPW method yielded an direct band gap of $2.5 \mathrm{eV}$ which is lower the 
experimentally reported value of $3.2 \mathrm{eV}$ for $\mathrm{SrTiO}_{3}$. The origin of this discrepancy may be the GGA. The nine valence bands at the $\Gamma$-point are the three triply degenerate level $\left(\Gamma_{15}, \Gamma_{25}\right.$ and $\left.\Gamma_{15}\right)$ separated by energies of $1 \mathrm{eV}$ $\left(\Gamma_{25}-\Gamma_{15}\right)$ and $1.6 \mathrm{eV}\left(\Gamma_{15}-\Gamma_{25}\right)$. The crystal field and the electrostatic interaction between oxygen $2 p$ orbital produce these splitting. In the conduction band the triply $\left(\Gamma_{25^{\prime}}\right)$ and doubly $\left(\Gamma_{12}\right)$ degenerate levels represent $t_{2 g}$ and $\mathrm{e}_{\mathrm{g}}$ states of Ti $3 \mathrm{~d}$ orbital separated by energy of $2.3 \mathrm{eV}$. A visual comparison of our band structure results with the result of reference [23] on $\mathrm{SrTiO}_{3}$ shows direct band gap at the $\Gamma$ point using the first-principles tight-binding linearized muffin-tin orbital (TB-LMTO) method $(1.4 \mathrm{eV})$ lower than by the FP-LAPW method. The zero of the energy was set at the top of the valence band and the energy scale is in $\mathrm{eV}$. The electronic structure in Figure 2 was calculated using the experimental lattice constant (a).The bottom of the valence band is mainley contribution of from $\mathrm{Sr} 3 p$ orbitals. The bands at around $-16 \mathrm{eV}$ originate from $\mathrm{O} 2 \mathrm{~s}$ orbitals. Nine valence bands near Fermi level between 0.0 and $4.7 \mathrm{eV}$ are derived from $\mathrm{O} 2 \mathrm{p}$ orbitals. It is to be noted that in the case of $\mathrm{BaTiO}_{3}$ the Ba $5 \mathrm{p}$ state and in $\mathrm{SrTiO}_{3}$ the $\mathrm{Sr} 4 \mathrm{p}$ state appear at higher energy than $\mathrm{O} 2 \mathrm{~s}$ state whereas in the case of $\mathrm{CaTiO}_{3}$ the $\mathrm{O} 2 \mathrm{~s}$ state appears at higher energy than $\mathrm{Ca} 3 p$ state. The band gap value for $\mathrm{SrTiO}_{3}$ in the cubic phase calculated in this work, and the values obtained by other methods are summarized in Table 4.

\subsection{Density of States}

The electron distribution in an energy spectrum is de- scribed by the density of states (DOS) and can be measured in photoemission experiments [24]. The total DOS spectrum of cubic $\mathrm{SrTiO}_{3}$ is shown in Figure 4, the valence and conduction band edges near the Fermi energy are quite sharp. This is consistent with the experimental

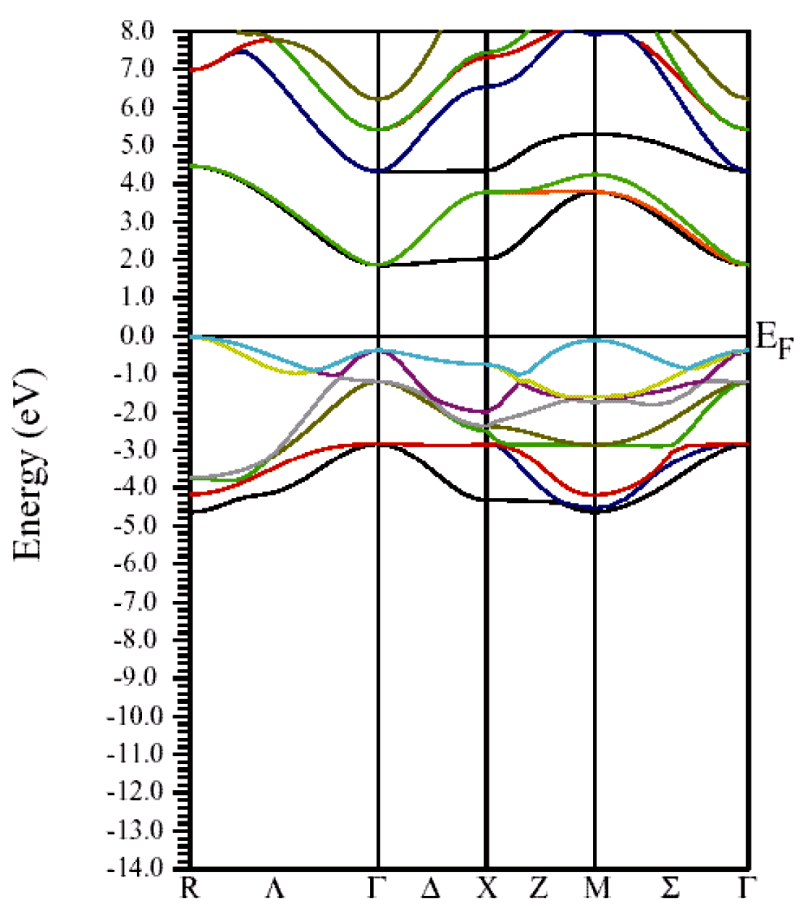

Figure 3. The calculated electronic energy-band structure of the Cubic $\mathrm{SrTiO}_{3}$. The zero of the energy was set at the top of the valence band.

Table 4. The results for the band gap calculated by this method and others.

\begin{tabular}{cccccc}
\hline & $\begin{array}{c}\text { FP-LAPW, GGA96 } \\
\text { With Theoretical } \\
\text { Constant }\end{array}$ & $\begin{array}{c}\text { FP-LAPW, GGA96 } \\
\text { With experemintal } \\
\text { Constant }\end{array}$ & $\begin{array}{c}\text { FP-LAPW, GGA91 } \\
\text { With Theoretical } \\
\text { Constant }\end{array}$ & $\begin{array}{c}\text { FP-LAPW, GGA91 } \\
\text { With experemintal } \\
\text { Constant }\end{array}$ & $\begin{array}{c}\text { FP-LAPW, LDA } \\
\text { With Theoretical } \\
\text { Constant }\end{array}$ \\
\hline $\begin{array}{c}\text { Typical Gap } \\
\text { Band Gap(eV) }\end{array}$ & $\Gamma$ & $\Gamma$ & $\Gamma$ & $\Gamma$ & $\Gamma$ \\
Difference with experi- \\
mental (0/0)
\end{tabular}


finding of a relatively sharp absorption edge in optical measurements of $\mathrm{SrTiO}_{3}$. Another very useful piece of information to examine is the hybridization and charge distribution

Figure 4 shows the total density of states for the valence and conduction band.In this figure the zero of the energy scale (the top of the valence band) shows the position of the Fermi level. To obtain a measure of the contribution of different atomic states in the band structure and also their possible hybridizations I made a detailed study of the partial density of the states. The study of the partial density of states showed that the hybridization of Ti $3 \mathrm{~d}$ and oxygen $2 \mathrm{p}$ and their contribution to the states on the valence and conduction band. There is, however, only a very weak hybridization of the $\mathrm{Sr} \mathrm{P}$ state with the O $2 p$ state in the valence band. The major contribution around the conduction band edge is from the Ti $3 \mathrm{~d}$ states with a small component from the $\mathrm{O} 2 \mathrm{p}$ states. The partial density of states (PDOS) of the $\mathrm{Sr}, \mathrm{Ti}$, and $\mathrm{O}$ atoms are shown in Figure 5. Where the low-energy peak at around -4 to $-1 \mathrm{eV}$ is a contribution mainly from the $\mathrm{O}$ $2 \mathrm{p}$ states with a small component from Ti $\mathrm{p}$ and $\mathrm{Sr} p$ orbitals. The valence states from $-4.7 \mathrm{eV}$ up to the Fermi energy are dominated by the $\mathrm{O} 2 \mathrm{p}$ states and strongly hybridized with the $\mathrm{Ti} 3 \mathrm{~d}$ state. It should be pointed out that the PDOS spectrum in Figure 5 includes one $\mathrm{Sr}$, one $\mathrm{Ti}$ atom, and three $\mathrm{O}$ atoms. Therefore the height of the O $2 p$ DOS peak is much higher than that of the Ti $3 d$ states.There exists a $\mathrm{p}-\mathrm{d}$ hybridization as is evident from this figure.

A quick estimate suggest that there is only a very weak hybridization of the $\mathrm{Sr} p$ state with the $\mathrm{O} 2 \mathrm{p}$ state, but there is a very strong hybridization betIen Ti $3 p$ and O $2 p$ state in the valence band. This means that this system is not quite an ionic bond but it has rather a large covalency. There exists a p-d hybridization as is evident from this fiogure. The $\mathrm{Ti} 3 \mathrm{~d}$ contribution is zero at the valence band maximum but rises with increasing binding energy. Conversely the $\mathrm{O} 2 \mathrm{p}$ contribution rises from zero at the conduction band minimum with increasing energy. This reflects the Ti $3 d-O 2 p$ covalency.

\subsection{Electronic Charge Density}

This is shown in Figure 6 for (110) plane. The electron density distribution indicated that the bond between $\mathrm{Sr}$ and $\mathrm{TiO}_{2}$ is ionic while between the $\mathrm{Ti}$ and $\mathrm{O}$ is covalent. To further study the charge distribution and also the nature of the chemical bonding, I calculated the electron charge density for the cubic phase of $\mathrm{SrTiO}_{3}$. The distribution of charge around the $\mathrm{Sr}$ site indicates that the bonding between $\mathrm{Sr}$ and $\mathrm{TiO}_{2}$ is mainly ionic. Further, the interatomic distance between the $\mathrm{Ti}$ and $\mathrm{O}$ is only $3.69 \mathrm{au}$ and that between $\mathrm{Sr}$ and $\mathrm{O}$ is $5.22 \mathrm{au}$ indicating that the bonding between $\mathrm{Ti}$ and $\mathrm{O}$ is covalent in nature. The effective charge density of cubic $\mathrm{SrTiO}_{3}$ was computed using the calculated electron wave function and density. The results are shown in Figure 6. Figure 6(a) shows the charge density in a (110) plane through $\mathrm{Ti}, \mathrm{Sr}$, and $\mathrm{O} 1$ atoms. In addition to the electron charge density in real space for the (100) plane that passes through $\mathrm{Ti}$ and $\mathrm{Sr}$ atoms is shown in Figure 7.

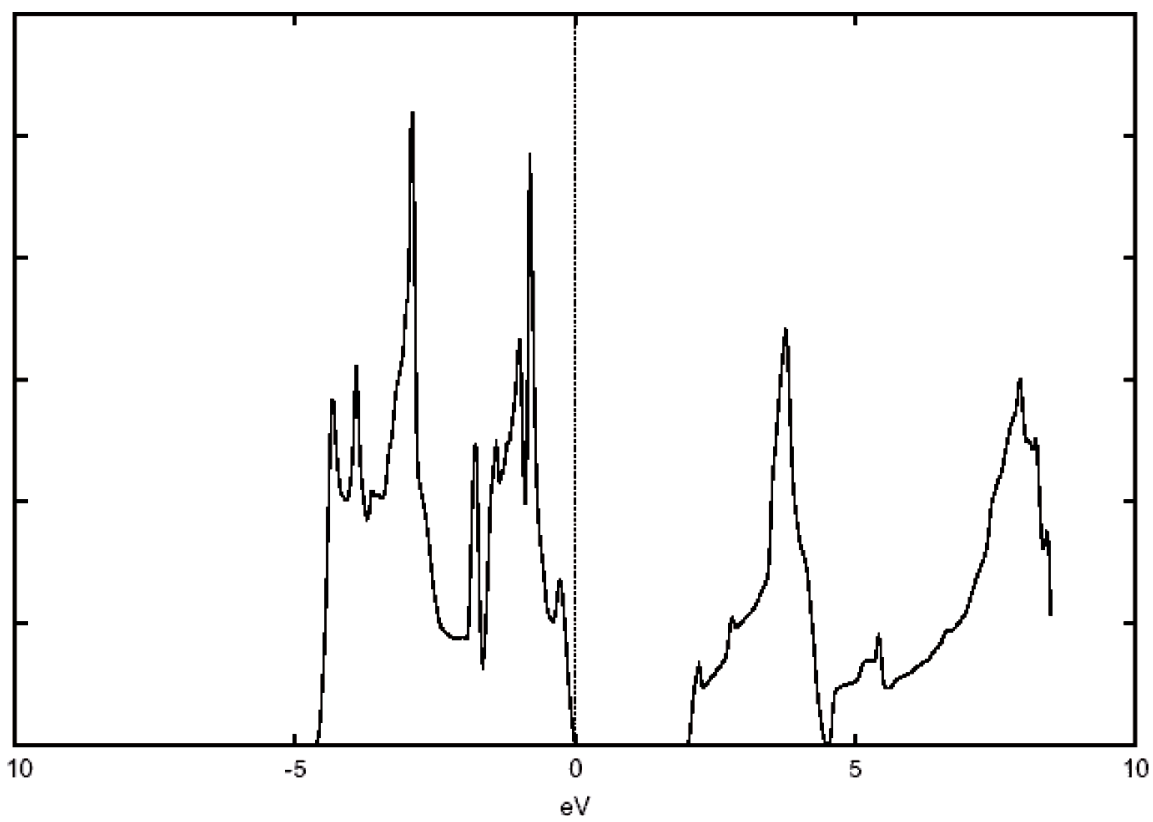

Figure 4. The total density of states (DOS) for Cubic $\mathrm{SrTiO}_{3}$. 


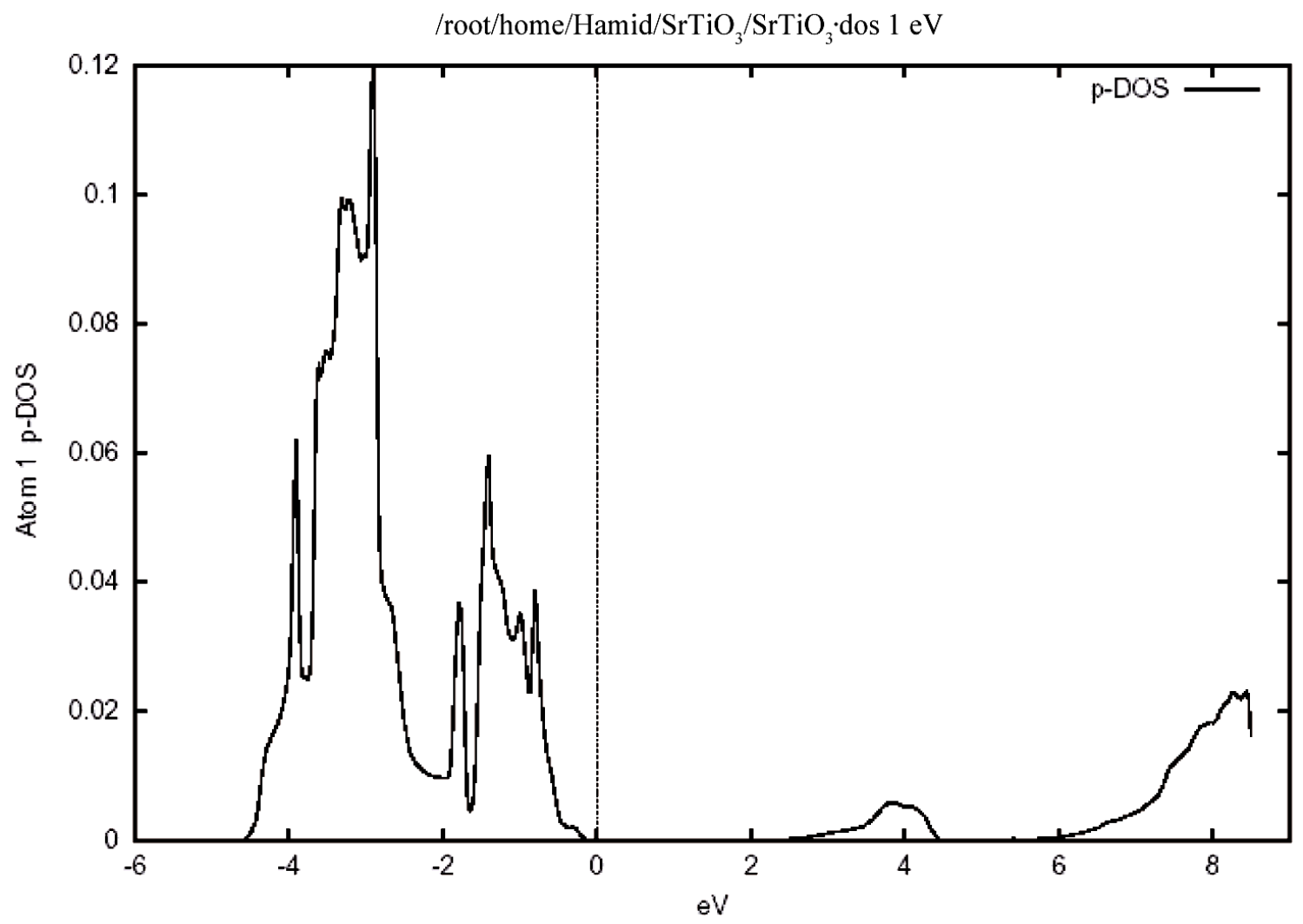

(a) (i)

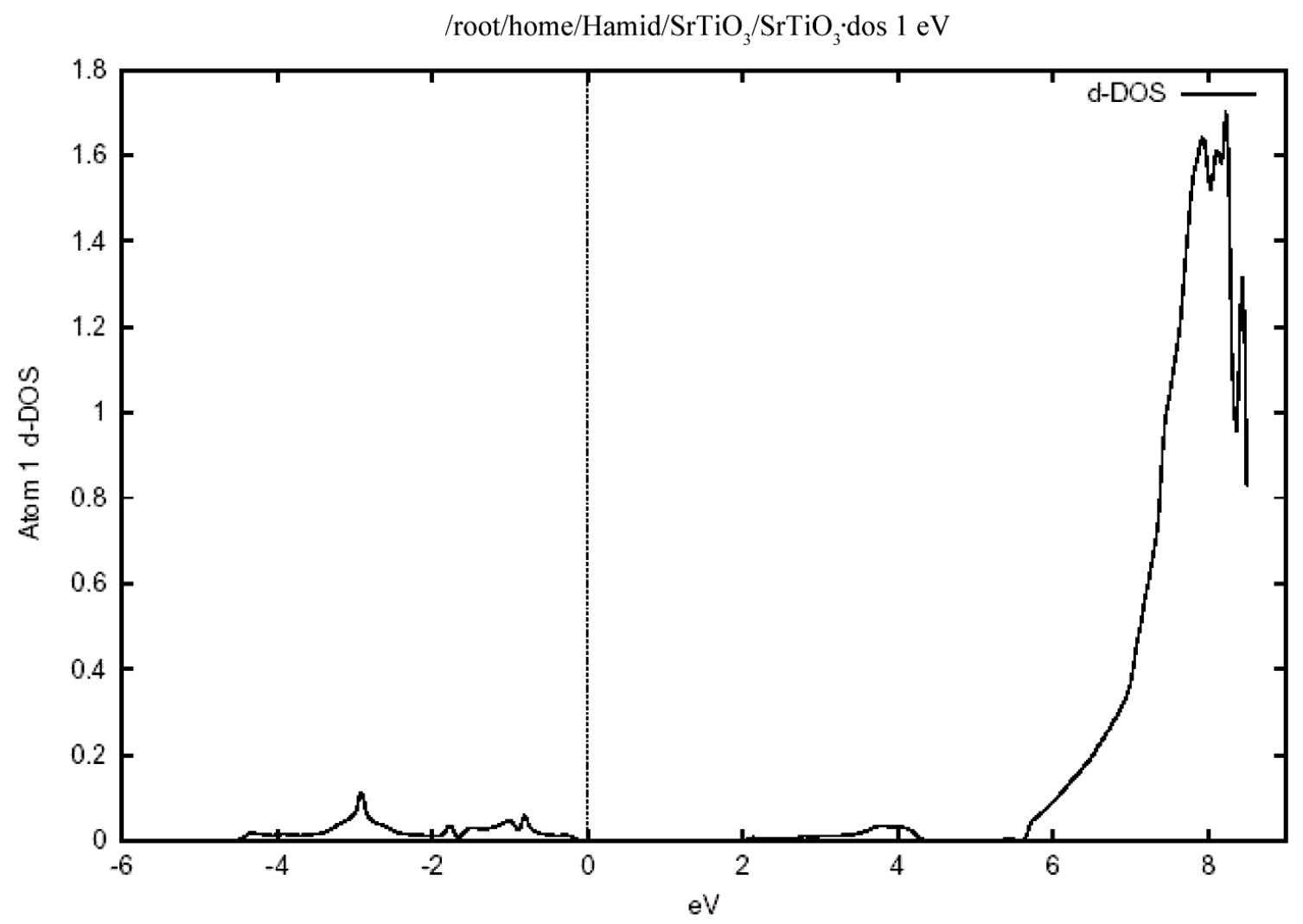

(a) (ii) 


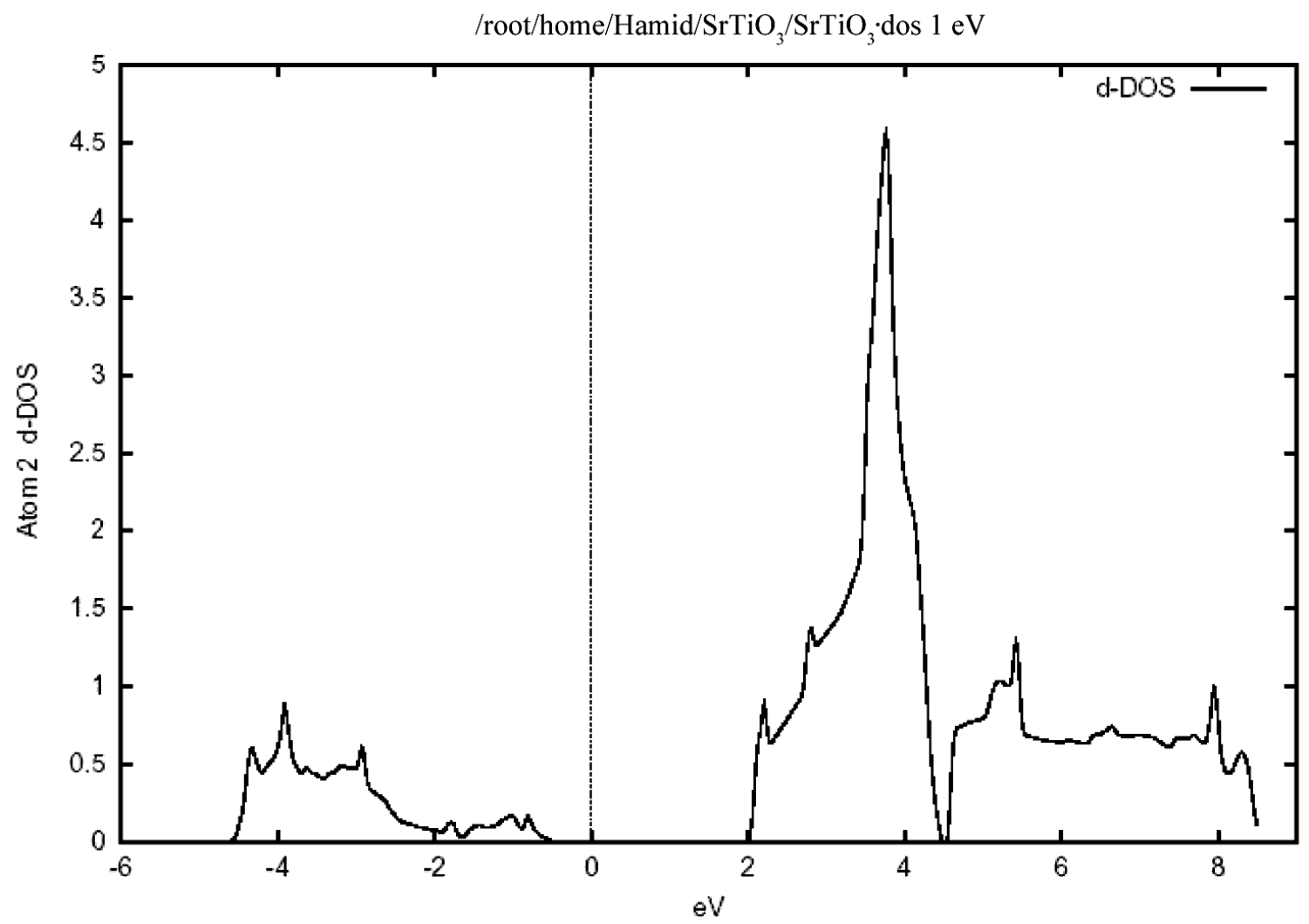

(b) (i)

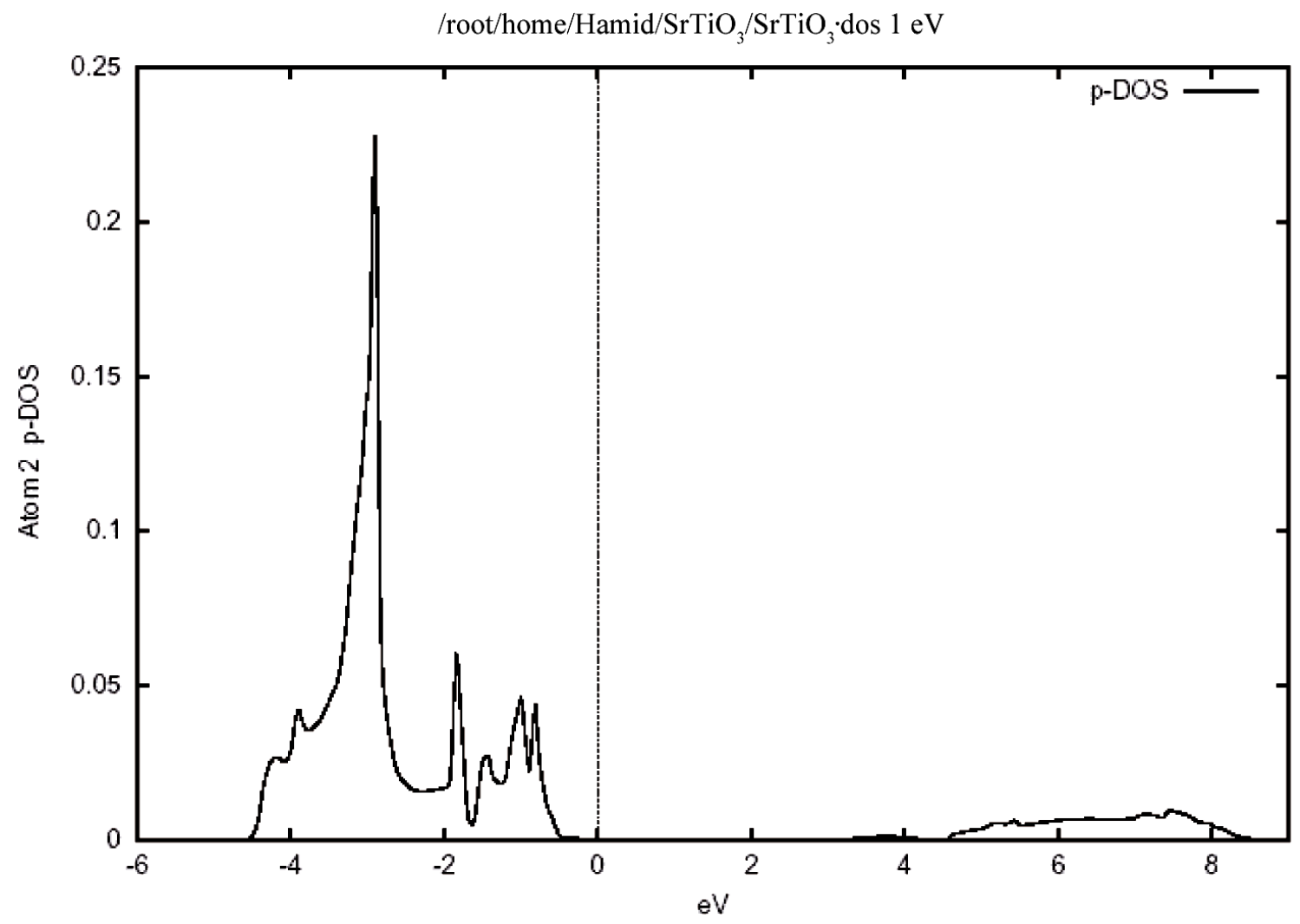

(b) (ii) 


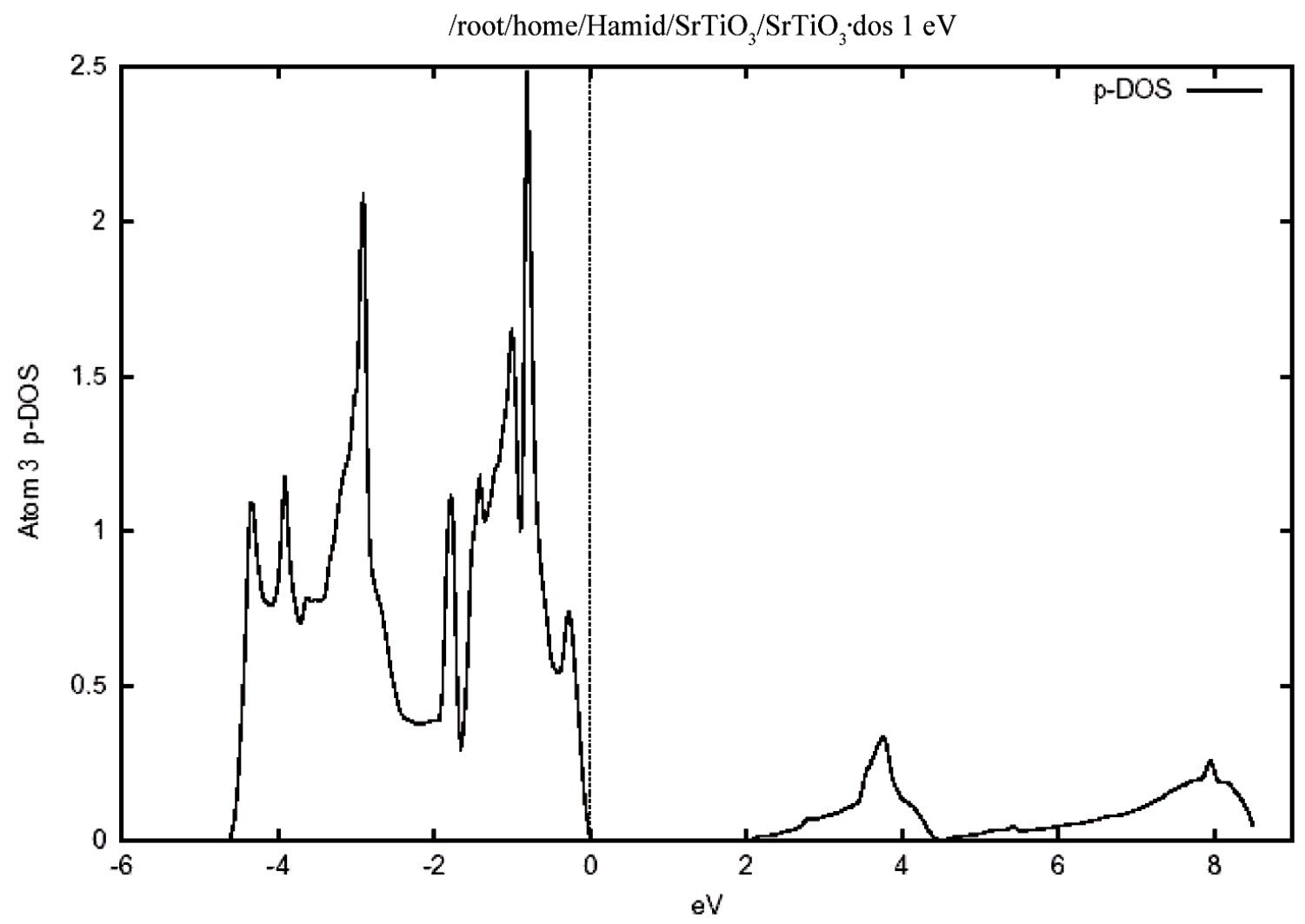

(c)

Figure 5. The partial densities of states (PDOS) showing the contributions of $\mathrm{Sr}$, $\mathrm{Ti}$ and $\mathrm{O}$ atoms. One $\mathrm{Sr}$, one $\mathrm{Ti}$ and three $\mathrm{O}$ atoms from one unit cell of cubic $\mathrm{SrTiO}_{3}$ are included in the calculation. (a) Density of states for (i) p orbital, (ii) d orbital atom Sr. (b) Density of states for (i) d orbital, (ii) p orbital atom Ti. (c) Density of states for p orbital atom 0.

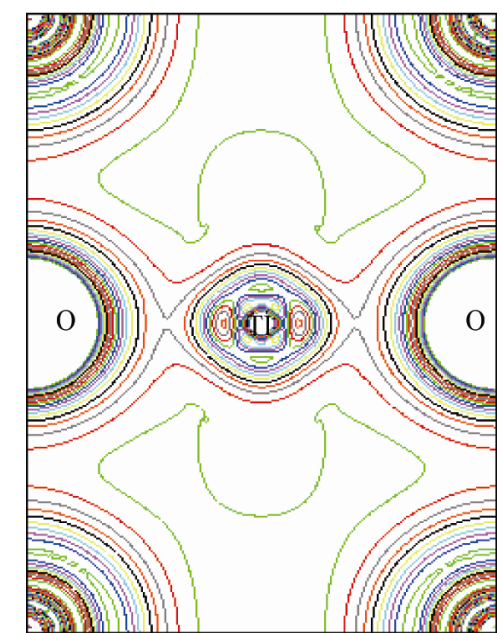

(a)

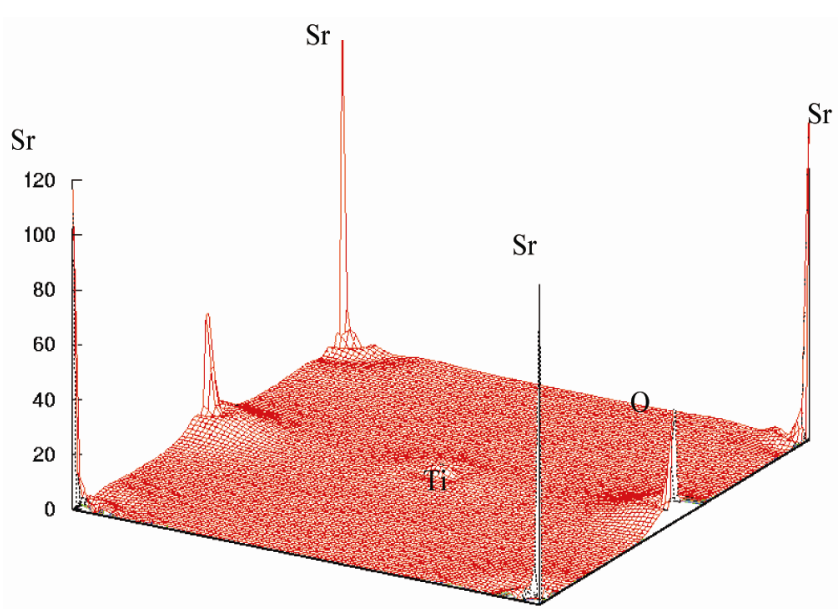

(b)

Figure 6. (a) The electron-density in (110) plane, (b) in three dimension.

The Ti-O bond, however has covalent character, this is quite apparent from the noticeable and $\mathrm{TiO}_{2}$ is ionic while between the $\mathrm{Ti}$ and $\mathrm{O}$ is covalent.charge distribution at the middle of the Ti-O bond. The charge distribu- tions in Figure 7(a) are consistent with the reported results of reference [23]. The study of Figures 6 and 7 shows that there is the important hybridization between Ti $3 d$ and O $2 p$ which again emphasizes the large cova- 


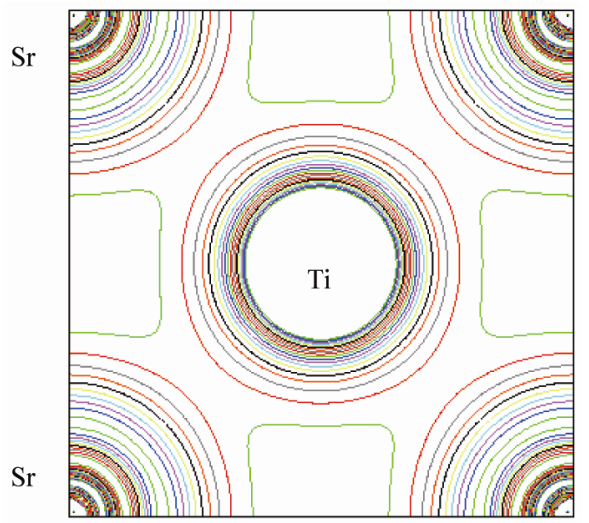

(a)

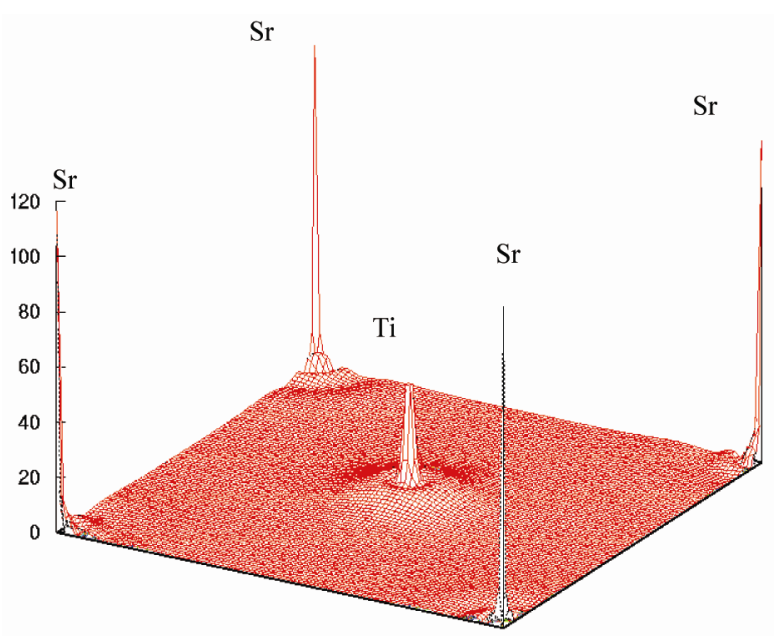

(b)

Figure 7. Electron density distribution for $\mathrm{SrTiO}_{3}(\mathrm{a})$ in the (100) plane and (b) in three dimension.

lent character of their bond.

\section{Conclusions}

I have made a detailed investigation of the electronic structure, band structure and DOS of perovskite $\mathrm{SrTiO}_{3}$ using the FP-LAPW method. The total DOS obtained from my first principles calculations are compared with experimental results [20]. It has been concluded that the top of the volence band is mainly composed of the $\mathrm{O} 2 \mathrm{p}$ orbitals in non-bonding states and the lower part of the $\mathrm{VB}$ is formed by bonding states between the Ti $3 \mathrm{~d}$ and $\mathrm{O}$ $2 p$ orbitals. The CB mainly consists of $3 d$, 4 sand $4 p$ states of $\mathrm{Ti}$ and $3 \mathrm{~d}$ and $4 \mathrm{~s}$ state of Sr. The calculations show that the fundamental gap of $\mathrm{SrTiO}_{3}$ is direct at the $\Gamma$ point. My calculated fundamental gap $2.5 \mathrm{eV}$ is smaller than the experimentally reported value $3.2 \mathrm{eV}$, which the origin of this discrepancy may be the GGA . The chemical bonding of $\mathrm{SrTiO}_{3}$ is also analyzed. The $\mathrm{TiO}_{2}$ complex is bonded mainly by covalent bonds and the $\mathrm{Sr}$ and $\mathrm{TiO}_{2}$ constituents are bonded mainly ionically. The calculated results compared with the experimental results.

\section{References}

[1] M. E. Lines and A. M. Glass, "Principles and Applications of Ferroelectrics and Related Materials," Clarendon, Oxford, 1977.

[2] F. W. Lytle, "X-Ray Diffractometry of Low-Temperature Phase Transformations in Strontium Titanate," Journal of Applied Physics, Vol. 35, No. 7, 1964, pp. 2212-2215. doi:10.1063/1.1702820

[3] R. Viana, P. Lunkenheimer, J. Hemberger, et al., "Dielectric Spectroscopy in $\mathrm{SrTiO}_{3}$," Physical Review B, Vol. 50, No. 11, 1994, pp. 601-605.
doi:10.1103/PhysRevB.50.601

[4] M. Cardona "Optical Properties and Band Structure of $\mathrm{SrTiO}_{3}$ andBaTiO 3 ," Physical Review, Vol. 140, No. 2A, 1965, pp. 651-655. doi:10.1103/PhysRev.140.A651

[5] D. Bauerle, W. Braun, V. Saile and G. Sprüssel, "Vacuum Ultraviolet Reflectivity and Band Structure of $\mathrm{SrTiO}_{3}$ and $\mathrm{BaTiO}_{3}$ " Zeitschrift für Physik B, Vol. 29, No. 3, 1978, pp. 179-184.

[6] O. M. Nes, K. A. Müller and T. Suzuki, "Elastic Anomalies in the Quantum Paraelectric Regime of $\mathrm{SrTiO}_{3}$ This Article Has Been Downloaded from IOPscience," Europhys. Lett, Vol. 19, 1992, p. 397.

[7] C. H. Perry, et al., "Infrared Studies of Perovskite Titanates," Physical Review, Vol. 135, No. 2A, 1964, pp. 408-412. doi:10.1103/PhysRev.135.A408

[8] J. L. Servoin, et al., "Infrared Dispersion in $\mathrm{SrTiO}_{3}$ at High Temperature," Physical Review B, Vol. 22, 1980, pp. 5501-5506. doi:10.1103/PhysRevB.22.5501

[9] K. A. Muller, et al., "Indication for a Novel Phase in the Quantum Paraelectric Regime of SrTiO3," Zeitschrift für Physik B, Vol. 48, 1991, pp. 277-283.

[10] K. A. Muller and H. Burkard, "SrTiO ${ }_{3}$ : An Intrinsic Quantum Paraelectric below 4 K," Physical Review B, Vol. 19, No. 7, 1979, pp. 3593-3602. doi:10.1103/PhysRevB.19.3593

[11] W. Zhong and D. Vanderbilt, "Effect of Quantum Fluctuations on Structural Phase Transitions in $\mathrm{SrTiO}_{3}$ and $\mathrm{BaTiO}_{3}$," Physical Review B, Vol. 53, No. 9, 1996, pp. 5047-5050. doi:10.1103/PhysRevB.53.5047

[12] W. G. Spitzer, R. C. Miller, D. A. Kleinman and L. E. Howarth, "Far Infrared Dielectric Dispersion in $\mathrm{BaTiO}_{3}$, $\mathrm{SrTiO}_{3}$, and $\mathrm{TiO}_{2}$," Physical Review, Vol. 126, No. 10, 1962, pp. 1710-1721. doi:10.1103/PhysRev.126.1710

[13] W. Jauch and A. Palmer, "Anomalous Zero-Point Motion in SrTiO3: Results from $\gamma$-Ray Diffraction," Physical Review B, Vol. 60, No. 5, 1999, pp. 2961-2963.

[14] P. A. Fleury, J. F. Scott and J. M. Worlock, "Soft Phonon 
Modes and the $110 \mathrm{~K}$ Phase Transition in $\mathrm{SrTiO}_{3}$," Physical Review Letters, Vol. 21, No. 1, 1968, pp. 16-19. doi:10.1103/PhysRevLett.21.16

[15] G. Shirane and Y. Yamada, "Lattice-Dynamical Study of the $110 \mathrm{~K}$ Phase Transition in $\mathrm{SrTiO}_{3}$," Physical Review, Vol. 177, No. 2, 1969, pp. 858-863. doi:10.1103/PhysRev.177.858

[16] J. P. Perdew, J. A. Chevary, S. H. Vosko, et al., "Atoms, Molecules, Solids, and Surfaces: Applications of the Generalized Gradient Approximation for Exchange and Correlation," Physical Review B, Vol. 46, No. 11, 1992, pp. 6671-6687. doi:10.1103/PhysRevB.46.6671

[17] J. P. Perdew, "Generalized Gradiant Approximation for Exchange and Correlation. A Look Forward and Backward," Physica B, Vol. 172, No. 1-2, 1991, pp. 1-6. doi:10.1016/0921-4526(91)90409-8

[18] R. D. King-Smith and D. Vanderbilt, "First-Principles Investigation of Ferroelectricity in Perovskite Compounds," Physical Review B, Vol. 49, No. 9, 1994, pp. 5828-5844. doi:10.1103/PhysRevB.49.5828

[19] R. D. King-Smith and D. vanderbilt, "A First-Principles
Pseudopotential Investigation of Ferroelectricity in Barium Titanate," Ferroelectrics, Vol. 136, 1992, pp. 85-94.

[20] P. G. Perkins and D. M. Winter, "Calculation of Band Structures and Electronic Properties from Models of $\mathrm{SrTiO}_{3}$," Journal of Physics C: Solid State Physics, Vol. 16, No. 18, 1983, pp. 3481-3492. doi:10.1088/0022-3719/16/18/018

[21] R. W. G. Wyckoff, "Crystal Structures,” 2nd Edition, Vol. 2, Chemical Catalog Company Incorporated, New York, 1964.

[22] P. Blaha and K. Schwarz, "WIEN2k," Vienna University of Technology, Vienna, 2004.

[23] S. Sonali, T. P. Sinha and M. Abhijit, "Structural and Optical Properties of Paraelectric $\mathrm{SrTiO}_{3}$," Journal of Physics: Condensed Matter, Vol. 12, No. 14, 2000, p. 3325. doi:10.1088/0953-8984/12/14/309

[24] D. Bagayoko, G. L. Zhao, J. D. Fan and J. T. Wang, “Ab Initio Calculations of the Electronic Structure and Optical Properties of Ferroelectric Tetragonal $\mathrm{BaTiO}_{3}$," Journal of Physics: Condensed Matter, Vol. 10, No. 25, 1998, pp. 5645-5655. doi:10.1088/0953-8984/10/25/014 